\title{
La evolución de la industria de autopartes en Querétaro, 1993-2008
}

\section{The evolution of the auto parts industry in Querétaro, 1993-2008}

\author{
Selva L. Daville-Landero*
}

\begin{abstract}
From the 1990's on, and specifically from 1994 when the North American Free Trade Agreement (NAFTA) first came into force, the policy of reorienting the Mexican economy toward the outside world was stepped up. This article discusses the changes that have taken place in the auto parts industry established in the State of Queretaro, based on existing data from the Census and evidence collected from a survey. Some of the major findings from this research include an increasing concentration of industrial activity and of the auto parts branch in the metropolitan area of Queretaro. A greater prevalence of transnational companies'subsidiaries was also observed, which has brought about the virtual disappearance of local productive chains.
\end{abstract}

Keywords: industrial restructuration, automotive industry, auto parts industry.

\section{Resumen}

A partir de los ańos noventa, y específicamente desde 1994 con la firma del Tratado de Libre Comercio de América del Norte (TLCAN), se intensificó la política de reorientación de la economía mexicana hacia el exterior. En este artículo se analizan los cambios ocurridos en la industria de autopartes establecida en el estado de Querétaro con base en la información censal y la recabada mediante una encuesta. Una de las principales conclusiones de este estudio es el reforzamiento de la concentración de la actividad industrial y de la rama de autopartes en la Zona Metropolitana de Querétaro. También se pudo observar un mayor predominio de las filiales de las trasnacionales, que ha traído como consecuencia, prácticamente, la desaparición de encadenamientos productivos locales.

Palabras clave: reestructuración industrial, industria automotriz, industria de autopartes.

* Universidad Nacional Autónoma de México, México. Correo-e: daville@servidor.unam.mx. 


\section{Introducción}

El trabajo que aquí se presenta tiene como objetivo analizar los cambios ocurridos en la industria de autopartes de Querétaro y su efecto territorial, en el contexto de la intensificación de la apertura comercial con la firma, en 1994, del TLCAN, así como de la búsqueda de adaptación al modelo de producción de Toyota, el cual se ha constituido en el nuevo paradigma industrial en sustitución del fordismo y su producción en masa indiferenciada.

El enfoque teórico con el que se analizan las transformaciones ocurridas en la industria de autopartes y su desplieque territorial, es el de las cadenas globales de mercancías, desarrollado por Hopkins y Wallerstein (1994), y por Lee y Cason (1994) para el estudio específico de la cadena automotriz.

Las principales hipótesis con las que se trabajó son que la mayor apertura comercial y el proceso de mundialización tendrían como resultado el reforzamiento de la concentración industrial en los municipios tradicionalmente más importantes, debido a que allí se concentran las mejores condiciones generales para la producción.

Por otra parte, este mismo proceso daría origen a la destrucción de los encadenamientos productivos locales por la llegada y mayor importancia del papel de las filiales de grandes empresas trasnacionales de la rama de autopartes; las cuales se rigen bajo la lógica de su casas matrices y funcionan en buena medida como enclaves, al importar casi la totalidad de sus insumos y orientarse predominantemente hacia el exterior.

En un primer acercamiento, el análisis se basó en la información contenida en los censos industriales correspondientes, generados por el Instituto Nacional de Estadística, Geografía e Informática (INEGI). Posteriormente, con el propósito tener información acerca de los vínculos industriales que establecen las empresas, se llevó a cabo una encuesta entre las de la industria de autopartes localizadas en Querétaro, cuya muestra aleatoria estratificada está integrada por 13 empresas.

En el primer apartado se plantean los elementos de análisis asumidos por el enfoque de las cadenas globales de mercancías. En el segundo se presentan las estadísticas que muestran las características generales de la industria en el estado de Querétaro, donde se destaca a nivel de subsectores la metal-mecánica, y a nivel de rama la industria automotriz, representada en Querétaro por la producción de autopartes, al no existir una planta ensambladora en la entidad. De igual manera, con los datos estadísticos se da cuenta de la localización de la industria manufacturera en su conjunto, así como de la de autopartes. En tercero se analiza la información obtenida a partir de la encuesta levantada entre las empresas de 
la industria de autopartes. Finalmente se presentan las conclusiones en relación con la evolución de la industria de autopartes durante el periodo estudiado.

\section{El enfoque de las cadenas globales de mercancías}

Como se mencionó, el proceso de industrialización ha experimentado cambios muy importantes en las tres últimas décadas. La producción y exportación de manufacturas se localiza en países tanto del centro (caracterizados por tener gran riqueza, una producción sofisticada y diversificada, y Estados fuertes) como de la periferia capitalista (con poca riqueza, una producción basada en bajos salarios y Estados débiles) (Goldfrank, 1995).

Entre estos cambios destaca la concepción de la fábrica global, en la cual la producción de una mercancía abarca a varios países, y a cada uno de ellos le corresponde desempeñar las labores en las cuales obtendría una ventaja de costos. Por ejemplo, durante la década de los años noventa, los componentes de un Ford Escort eran fabricados y ensamblados en 15 países alrededor de tres continentes. De esta manera, el capitalismo actual desagrega segmentos de la producción y el consumo rebasando las fronteras nacionales, bajo la estructura organizacional de redes densas de firmas o empresas.

El enfoque de las cadenas globales de mercancías permite analizar las características espaciales de dichas transformaciones a través de la economía-mundo, así como de las relaciones que constituyen dichos procesos. El objetivo central que se plantea esta visión es entender los cambios ocurridos en la organización espacial de la producción y el consumo en la economía capitalista contemporánea.

Hopkins y Wallerstein definen una cadena de mercancías como "una red de procesos de trabajo y producción cuyo resultado final es una mercancía terminada" (1986: 159). En esta concepción, una cadena global está integrada por sets de redes interorganizacionales agrupadas alrededor de una mercancía o producto, vinculando hogares, empresas y Estados con otros dentro de la economía-mundo. Estas redes se caracterizan porque estan específicamente situadas, construidas socialmente e integradas localmente, subrayando la inserción (embeddedness) social en la organización económica.

Los procesos específicos o segmentos dentro de una cadena de mercancías se pueden representar como cajas o nodos, vinculados conjuntamente en redes. Cada nodo sucesivo dentro de una cadena de mercancías involucra la adquisición y/o la organización de insumos, por ejemplo, materias primas o productos semiterminados, fuerza de trabajo (y su 
aprovisionamiento), transporte, distribución (vía mercados o transferencias) y consumo.

Otra ventaja del enfoque de las cadenas globales de mercancías es el análisis de las desigualdades espaciales que existen en la economía-mundo en términos de accesos diferenciados a los mercados y a los recursos. Este enfoque también permite establecer los vínculos macro-micro que existen entre procesos que, tradicionalmente, se considera que están contenidos en unidades de análisis globales, nacionales y locales. De igual manera permite profundizar en el análisis de la estructura y el cambio, tanto en la escala supra o infranacional de la economía mundo.

\subsection{Las cadenas en la industria del automóvil}

Un ejemplo de los cambios ocurridos en los últimos años es el de algunos países de la semiperiferia que han avanzado y escalado en la jerarquía del sistema-mundo. Estos países han utilizado una tecnología más avanzada y están produciendo bienes con alto valor agregado, modificando relativamente la división internacional del trabajo tradicional. Sobre todo en la industria automotriz se puede observar este proceso con mayor claridad. Esta industria se caracteriza porque es intensiva en capital y tecnología, con altas barreras de entrada, por lo que se pensaba que los países recién industrializados, que tienen gran oferta de trabajo, no tendrían una ventaja comparativa en industrias de este tipo. No obstante, algunos países semiperiféricos empezaron a exportar en estas industrias y han tenido éxito al penetrar en mercados internacionales (Lee y Cason, 1994: 223).

Los casos de mayor éxito en las exportaciones automotrices son Corea del Sur, México y Brasil, que registraron un rápido crecimiento en sus exportaciones durante los años ochenta. En estos tres países las exportaciones del sector automotriz representaron, en 1988, una proporción significativa de las exportaciones totales, $11.1 \%$ en el caso de Brasil, $17.1 \%$ en México y 6.5\% en Corea del Sur.

Una cuestión interesante a destacar en el estudio del proceso de reestructuración industrial, es que la misma industria está asociada a diferentes patrones de desarrollo y da cuenta de la heterogeneidad de dicho proceso. Los países de Latinoamérica presentan una dominación casi total de la industria por corporaciones trasnacionales, mientras que Corea del Sur está bajo el dominio de capital local. El perfil de exportación también es diferente, Corea del Sur y, en menor medida, Brasil se han orientado a la exportación de vehículos terminados. Mientras que México, en un inicio, se dedicó a cubrir el nicho exportador de autopartes. Otra diferencia significativa entre los países es el destino de sus exportaciones. México y Corea del Sur envían la mayor parte de sus exportacio- 
nes al mercado estadounidense, mientras que Brasil tiene un rango más amplio de clientes, con exportaciones significativas hacia Europa y el Medio Oriente.

Con el propósito de explicar estos diferentes patrones de desarrollo, Lee y Cason (1994: 225) analizan la cadena de mercancías de la industria automotriz, y concluyen que se pueden presentar factores explicativos como la política estatal, la estrategia de negocios y la geografía, los cuales influyen significativamente en los patrones de internacionalización de la industria automotriz. De igual manera, plantean que la cadena de mercancías es una herramienta poderosa para analizar las trayectorias de desarrollo y de movilidad ascendente de los países semiperiféricos.

\subsection{La aplicación del concepto de cadenas de mercancías}

Para Lee y Cason (1994), la literatura de los sistemas-mundo permite realizar un análisis de la estructura de la economía-mundo, pero consideran que pone menos énfasis en las trayectorias de desarrollo de los países específicos dentro del sistema. Por otra parte, la literatura centrada en el análisis del Estado ha hecho lo opuesto, ha sobrevalorado el papel de éste en la determinación de la especificidad de los países. Dado que cada país tiene una trayectoria histórica propia, a menudo es contraproducente enfatizar estas diferencias, mientras que se ignoran las fuerzas sistémicas que influyen en esta trayectoria.

De esta manera, dichos autores proponen establecer un puente entre ambos enfoques, centrándose en la cadena productiva automotriz de varios países y construir un marco útil para entender la dinámica de las industrias particulares y su relación con la economía internacional. Al analizar la cadena productiva se puede desagregar una industria en sus diferentes etapas, y ayuda a comprender cuáles son los factores más importantes que influyen en una trayectoria industrial. También permite enfatizar el dinamismo y analizar las posibilidades del escalamiento industrial. El avance en la escala industrial generalmente implica tener más control sobre algunas partes del proceso de producción, así como la capacidad para generar conocimiento técnico que será crucial en los proyectos posteriores de escalamiento.

Este enfoque también enfatiza la importancia del concepto de nicho de mercado, que implica diferentes tipos de integración con el mercado internacional. El nicho particular que ocupa un país dentro de una cadena productiva refleja las configuraciones nacionales tanto institucionales como económicas y sociales de cada país; al mismo tiempo, el nicho y la integración concomitante en la economía internacional se refleja en la economía y en la política locales. 
Como se mencionó, para Hopkins y Wallerstein una cadena de mercancías es "una red de procesos de trabajo y producción cuyo resultado final es una mercancía terminada" (1986: 159). El automóvil es, probablemente, una de las mercancías más complejas que se puedan analizar. La producción de un automóvil es resultado de un proceso industrial extremadamente intrincado que vincula proveedores y productores de varias partes del mundo. Lee y Cason (1994) no pretenden analizar la cadena completa de la industria automotriz, ni realizar un análisis detallado de la producción de autopartes, sino centrarse en las redes que se establecen entre los productores de autopartes y los ensambladores.

En este aspecto, el modelo desarrollado por Toyota se ha caracterizado por establecer una estrecha relación entre la ensambladora y sus proveedores desde el momento en que da inicio el desarrollo de un producto. Ambos comparten información sobre sus costos y métodos de producción; y en conjunto buscan mejorar la calidad y reducir costos. Bajo este sistema las armadoras trasladan parte sustancial de las tareas y de la responsabilidad en el diseño, la ingeniería detallada y la fabricación de diversas autopartes y componentes a sus proveedores (Womack et al., 1992: 135).

De acuerdo con Gereffi y Korzeniewicz (1990: 51), la cadena de mercancías se conforma por cuatro segmentos: 1) abastecimiento de materias primas, 2) producción, 3) exportación, y 4) marketing y ventas al menudeo (retailing). Este planteamiento lo modifican Lee y Cason, ya que analizan una industria muy compleja. En lugar de centrarse en el abastecimiento de materia primas, lo hacen en las redes de suministro. $\mathrm{Y}$ en lugar de separar la exportación, la incluyen tanto en las redes de suministro de partes como en las de marketing, debido a que la integración a la cadena global puede ocurrir en diferentes lugares.

Desde sus inicios la industria automotriz se ha orientado tanto a los mercados internos como externos. En la mayor parte de países periféricos se desarrolló durante la etapa de sustitución de importaciones; en este periodo se tenía como objetivo desarrollar la mayor parte de la cadena dentro de cada país, limitando los vínculos internacionales. En algunos casos, cuando la industria se orientó hacia la exportación, las cadenas de mercancías internas pudieron coexistir con la integración dentro de una cadena global.

En este sentido el caso de Corea es muy significativo, pues durante los años cincuenta y sesenta no tenía un papel significativo en la escena mundial, pero en los ochenta, específicamente en 1984, exportó 564,000 autos, mientras que en 1980 sólo había exportado 14,000 (Kim y Lee, 1994: 282-283). 


\subsection{Las redes de suministro de autopartes}

La producción y el suministro de autopartes son actividades estratégicas en la cadena de la industria automotriz, ya que la calidad y el costo de las autopartes determina la competitividad de los vehículos terminados. La construcción de redes eficientes de proveedores que produzcan una amplia variedad de autopartes es una de las tareas más importantes para las empresas terminales, ya que cada vehículo está hecho con más de 15,000 autopartes. Dentro de esta compleja cadena existen diferencias significativas, pues componentes importantes, como los motores, los fabrican las ensambladoras, mientras que una gran proporción de autopartes son producidas por empresas autopartistas y por subsidiarias.

Los proveedores de autopartes difieren en tamańo y en términos de su vinculación con las ensambladoras. Normalmente, una ensambladora necesita organizar a varios cientos de empresas autopartistas, que tienen muchos más empleados que las terminales. De acuerdo con el tamaño se puede observar una división del trabajo, las grandes empresas producen partes importantes y sofisticadas para las ensambladoras, mientras que las empresas pequeñas producen partes menos importantes que posteriormente se incluyen en las otras autopartes más sofisticadas.

En el modelo desarrollado por Toyota existe una organización piramidal en la cual se encarga a un solo proveedor, llamado de primera fila, un componente completo; por ejemplo los asientos, con la responsabilidad de entregarlos directamente en la planta para sólo ser montados. En esta estructura, el proveedor de primera fila dispone de un equipo de proveedores de segunda fila, que a su vez involucran en el proceso a proveedores de tercera y cuarta filas (Womack et al., 1992).

Por otra parte, las tres industrias consideradas presentan modelos de organización muy diferentes en lo referente a su industria de autopartes y en cuanto a las redes de proveedores de las ensambladoras. En primer lugar, las tres tienen diferentes tipos de vínculos con el capital extranjero. La industria de autopartes coreana está controlada principalmente por firmas locales, mientras que el capital extranjero ha jugado el papel principal en la industria de autopartes en México. El caso de Brasil se sitúa en un punto intermedio entre las otras dos.

\subsection{Nichos de exportación y escalamiento industrial}

Como un resultado combinado de sus diferentes tipos de integración en la cadena global, las tres industrias automotrices crearon diferentes nichos de exportación. Uno de ellos se refiere a los segmentos de los mercados mundiales capturados por los diferentes países dentro de un sector indus- 
trial. También un nicho de exportación se debe considerar tanto en términos del destino de las exportaciones como del nicho del producto.

Por un lado, México y Corea han dirigido sus exportaciones en más de $80 \%$ hacia el mercado estadounidense. En contraste, Brasil tiene un abanico mucho más amplio de clientes para su industria automotriz. Los diferentes destinos para las exportaciones de México y Brasil son el resultado de las estrategias globales de los principales exportadores y su capacidad de marketing. La posición dominante del mercado de Estados Unidos en el caso mexicano es resultado de las estrategias globales de las Tres Grandes (Chrysler, Ford y General Motors) como los exportadores dominantes. Los dos principales exportadores de Brasil, Volkswagen y Fiat, han exportado vehículos terminados a Europa y Sudamérica.

A pesar de las diferencias surgidas a partir de las estrategias seguidas por las empresas dentro de los países, las tres industrias automotrices han desarrollado nichos distintivos de producto. Las exportaciones mexicanas, hacia 1989, estaban mucho más concentradas en los sectores de autopartes y motores (más de $70 \%$ del total), mientras que las empresas coreanas exportaban más de $80 \%$ en el sector de vehículos terminados. De los tres países, Brasil tenía la mezcla de productos más diversificada en sus exportaciones, con niveles de exportación significativos en autopartes, motores y vehículos terminados. Posteriormente las tres industrias automotrices han tratado de escalar sus nichos de exportación.

La industria automotriz mexicana ha experimentado un escalamiento sustancial. Durante la segunda mitad de los años ochenta, las Tres Grandes incrementaron sus exportaciones de vehículos terminados; la proporción de éstos en las exportaciones totales alcanzó $42 \%$ de las exportaciones del sector automotriz en 1989; incremento que se relaciona con el cambio ocurrido en las estrategias globales de las Tres Grandes. Esta característica se fortaleció hasta alcanzar $66.18 \%$, en 2003 , mientras que los motores representaron 5.62\% y las autopartes 28.20\% (Juárez, 2005: 128).

Esta tendencia fue producto de importantes montos de inversión extranjera directa efectuada tanto en la industria terminal como en la de autopartes, entre 1994 y 2002 se registró un monto acumulado de 11,000 millones de dólares, teniendo su origen principalmente en países de Norteamérica (Estados Unidos 50.4 y Canadá 6.8\%), seguidos de Japón con 26.5, Alemania 8.2 y España 2.3\% (Mortimore y Barron, 2005: 19).

\section{La industria metal-mecánica en Querétaro}

El estudio del proceso de industrialización en Querétaro, donde el surgimiento de la industria metal-mecánica es el rasgo más distintivo del proceso industrializador de los años sesenta, tiene como base la concepción 
del territorio no sólo como una realidad física o administrativa, sino como producto de la acción humana que implica una dimensión socioeconómica y, por tanto, no es homogéneo. Un territorio concebido así no surge de manera espontánea, sino que emerge mediante un proceso histórico en el cual las instituciones influyen de manera significativa en la trayectoria de desarrollo que se ha seguido (Benko y Demazière, 2000).

Concebidos así, los territorios en su devenir histórico atraviesan por diferentes formas de desarrollo que dependen del grado de coherencia de su tejido económico y de las relaciones desarrolladas entre los diferentes actores. La concepción dinámica de la vida de un territorio requiere considerar el espacio dado (genealógico) y el espacio proyectado (la estructura activa), los cuales conforman la dimensión espacial de las relaciones sociales, las ya establecidas y las que se están desarrollando. De la interacción de ambas nace la realidad de una geografía socioeconómica (Benko y Lipietz, 2000).

El proceso de transformación del territorio de Querétaro de una entidad basada en las actividades agropecuarias hasta convertirse en un centro industrial de importancia, es un producto histórico-social en cuya construcción han intervenido diferentes actores. Los más dinámicos han sido el gobierno, en los ámbitos federal y local, y la iniciativa privada tanto nacional como extranjera, quienes con su acción conjunta han logrado consolidar un ambiente propicio y atractivo para la localización industrial, a pesar de recesiones mundiales, cambios en el modelo de desarrollo económico y crisis sexenales.

La acción gubernamental se orientó fundamentalmente a crear condiciones generales para la producción desarrollando zonas y parques industriales, así como modernizando la carretera México-Querétaro, con lo cual se elevó la rentabilidad de las inversiones industriales. El otro eje importante de la acción gubernamental estuvo conformado por políticas de exención fiscal.

En términos generales se puede decir que las políticas de fomento industrial han tenido buenos resultados, ya que no se han registrado pérdidas absolutas de empleo industrial y se ha logrado atraer inversión tanto nacional como extranjera. Los sectores industriales a los que se ha dirigido han estado determinados por las diferentes etapas del proceso de sustitución de importaciones, donde predominaron en un inicio las inversiones en sectores tradicionales como el textil y los vinculados al sector agropecuario, como el de alimentos. Posteriormente se impulsó la industria metal-mecánica (maquinaria y equipo), aunque cabe aclarar que ni en lo nacional ni en lo local se logró sustituir la importación de bienes de capital, quedando trunco el modelo. 
En los últimos 20 años, a partir de la apertura comercial, la firma del TLCAN y con el cambio de modelo económico orientado a las exportaciones, se dio inicio a la llamada modernización o reconversión industrial, frente a la cual este territorio industrial se ha encontrado ante el nuevo reto de lograr una competitividad que le permita insertarse en el mercado externo y enfrentar la competencia en el interno.

La industria tradicional de Querétaro tuvo sus inicios a fines del siglo XIX y principios del XX, cuando se establecieron empresas de la rama textil, como Hércules y La Purísima. Sin embargo, es hasta fines de la década de los cuarenta y principios de los cincuenta cuando tiene lugar un proceso de industrialización caracterizado por la transición de la manufactura textil hacia la industria alimentaria.

En este periodo se localizan varias empresas incentivadas tanto por las políticas gubernamentales que, en la etapa de la sustitución de importaciones fomentaron el crecimiento industrial, como por la construcción de las carreteras que unieron la ciudad de México con Querétaro, vía Ixmiquilpan y vía Toluca.

Durante la gubernatura de Octavio S. Mondragón (1949-1955), en 1951 se expidió la Ley Número 93, llamada Ley de Fomento y Protección Fiscal a la Industria, a las Nuevas Construcciones, a las Obras de Regadío y a los Trabajos Forestales. En ella se estipularon mecanismos selectivos para la concesión de franquicias fiscales en lo referente al impuesto predial urbano para las construcciones industriales, y del impuesto sobre ingresos mercantiles en la parte proporcional que le correspondía al Estado. Estos estímulos variaban entre la exención total de impuestos hasta por 10 años y la exención de $25 \%$ hasta por cinco años; y estaban en función del monto del capital invertido, el número de empleos generados, los beneficios que recibiría la comunidad, todo esto en el marco de las industrias nuevas y necesarias; para los hoteles por el número de cuartos; por el valor de la construcción en casas habitación, y en cuanto a obras de riego por la cantidad de hectáreas.

Esta ley se constituyó en un mecanismo fiscal de gran aliento que atrajo inversión foránea y fomentó la industrialización de Querétaro, ya que bajo su normatividad se exentó de impuestos estatales a las empresas hasta los años ochenta (Miranda, 2005: 157-161).

Entre las empresas asentadas en esta época en el municipio de Querétaro se encuentran: Textiles La Concordia, en 1947; Molino de Trigo El Fénix, en 1950; Productos Carnation de México, en 1950, y Kellog's de México, en 1951 (González y González, 1992: 75). Donde, como se puede observar, fueron importantes las empresas de la rama alimentaria.

Durante la gestión del gobernador Juan C. Gorráez (1955-1961) el fomento industrial siguió enmarcado en la mencionada Ley Número 93, 
abocándose a la mejora de infraestructura carretera que permitiera un mayor impulso a la industrialización. De esta manera, en 1958 se inauguró el camino corto México-Querétaro, que situó a la capital del estado estratégicamente dentro de la red nacional de carreteras, comunicándola ágilmente con la ciudad de México y el norte del país.

Sin embargo, debido al intenso tráfico en pocos años devino insuficiente y se planteó la necesidad de ampliarla y convertirla en autopista. Así, continuando en la línea de mejorar la infraestructura carretera, el siguiente gobernador, Manuel González de Cosío 1961-1967, junto con importantes hombres de negocios locales, entre los que destaca Bernardo Quintana Arrioja (presidente de Ingenieros Civiles Asociados, ICA), propusieron al gobierno federal la construcción de la autopista México-Querétaro, que inició en 1966 y fue inaugurada por el entonces presidente de la República, Gustavo Díaz Ordaz, en 1969 (Miranda, 2005: 190-192).

De este modo, en los años sesenta inicia otra etapa del proceso de industrialización incentivado por la construcción de la autopista México-Querétaro, la creación de parques industriales y el crecimiento de la inversión extranjera. La importancia de esta última puede constatarse en la memorias del ex gobernador de la entidad Manuel González de Cosío:

\footnotetext{
Cuando tomé posesión como gobernador, nuestra entidad contaba con tan sólo 28 empresas. Es interesante mencionar que de éstas, únicamente cinco eran consorcios o compañías de capital extranjero. En cambio, las 32 nuevas industrias asentadas en nuestra ciudad durante mi mandato, forman parte de esta categoría. Esto indica que las compañías internacionales se mostraban cada vez más conscientes de las ventajas que les ofrecía Querétaro como localización industrial estratégica (cit. en Estrada, 1996: 38).
}

Entre las medidas implementadas durante su gestión destaca una serie de gestiones ante la federación para facilitar el establecimiento de las industrias, ante las empresas eléctricas para obtener reducciones en las tarifas, así como el trámite de permisos de importación y exportación tanto de maquinaria como de materias primas y productos terminados.

Parte de esta década correspondió también al gobierno de Juventino Castro Sánchez (1967-1973), quien se destacó por la creación del Comité para el Desarrollo Industrial del Estado de Querétaro, encabezado por Bernardo Quintana y quien jugaría un rol muy importante al llevar a cabo una labor de promoción de las ventajas que obtendrían en Querétaro las empresas que se encontraban localizadas en el Valle de México. Este comité logró articular eficientemente los intereses de los sectores involucrados en la industrialización: el gobierno estatal, los capitales locales, y el foráneo representado sobre todo por ICA (Miranda, 2005: 301-303). 
En este periodo comienza el establecimiento de las empresas de la rama metal-mecánica, que actualmente es la más importante. Entre estas empresas se encuentran: Industria de Hierro, s.A. (1963), Link Belt Speeder, S.A., Compacto, s.A. (1962), que en forma conjunta establecen un importante complejo productor de maquinaria pesada para la construcción. Transmisiones y Equipos Mecánicos (Tremec) (1964), productora de cajas de velocidades y transmisiones tanto para el mercado nacional como internacional de la industria automotriz; Amerance de México, s.A., fabricante de artículos de hule para la industria automotriz; Agromak (Massey-Ferguson) y Máquinas de Proceso, ambas en 1968.

En la acción pública estatal se encuentra la creación de la Zona Industrial de San Juan del Río, donde se ubicaron empresas del sector de alimentos, transformando productos agrícolas en jugos de frutas y vinos.

Cabe señalar que ICA, gran consorcio nacional de la construcción, jugó un papel importante no sólo en la construcción de la autopista, sino también al desarrollar la principal zona industrial, Parques Industriales, que cuenta con todos los servicios y áreas de vivienda para los trabajadores de las empresas. Asimismo, instala en Querétaro las mencionadas empresas Industria del Hierro y Tremec.

También de esta época data el inicio de la construcción, por parte del gobierno del estado, del Parque Industrial Benito Juárez, ubicado en la carretera a San Luis Potosí, el cual sería inaugurado más tarde, en 1972.

El sector industrial de Querétaro tuvo otro fuerte desarrollo a lo largo de la década de los setenta, impulsado por la política federal de desconcentración de las actividades económicas de la ciudad de México, la cual había elevado su participación en la industria nacional de 28.5\% en 1940 a 48\% en 1980 (Garza, 1985: 223). Esta acción de política federal se vio complementada en el ámbito local con la decisión del gobernador Juventino Castro de no aumentar el impuesto predial, urbano y rústico; así como por la venta de los terrenos del nuevo parque industrial (Benito Juárez) a precios realmente simbólicos, muy por debajo del precio comercial (Estrada, 1996: 41).

Esa misma década, pero ya durante la gestión del gobernador Antonio Calzada Urquiza (1973-1979), también es notable por la llegada de poderosos grupos industriales nacionales y extranjeros, entre los que se pueden mencionar: Vitro, del Grupo Monterrey; Celulosa, del Grupo Chihuahua; Celanese Mexicana, Radiología de México, Polynova, Melco, Mabe y Uniroyal. Mención especial merece el Grupo Spicer, ya que no instaló una sola empresa, sino seis; y la construcción del Complejo Querétaro de Fertilizantes Mexicanos, empresa paraestatal en sus inicios y que en la actualidad ya privatizada se llama Agromex. 
Como se puede observar, otra característica importante de la acción gubernamental consiste en que en Querétaro no privó la inversión productiva, sino que se orientó hacia la creación de condiciones generales para la producción que elevaran la rentabilidad de las inversiones y las hicieran factibles. El otro caso de inversión pública en producción es el de Turborreactores, inaugurada en 1982 y dedicada a la reparación de motores.

Con la década de los años setenta concluye una etapa económica y social importante, conocida como desarrollo estabilizador, durante la cual se logró un crecimiento sostenido, con baja inflación y estabilidad cambiaria, lo cual permitió, en términos generales, una mejora en las condiciones de vida de sectores importantes de la población nacional. Con esta etapa también concluyó la puesta en marcha de la política de sustitución de importaciones, que en México no alcanzó a consolidar plenamente la fase de bienes de capital, quedando trunca nuevamente la expectativa de desarrollo.

Los años ochenta han sido caracterizados como la década perdida para toda América Latina, y México no fue la excepción. En 1982 tuvo lugar una de las crisis sexenales de mayor impacto, derivada de la caída de los precios del petróleo, que trajo como consecuencia una nueva devaluación del peso frente al dólar, un acelerado crecimiento de la deuda externa y una fuga masiva de capitales.

La situación era muy difícil, el producto se contrajo $0.6 \%$, la inflación alcanzó $98.8 \%$, y las reservas internacionales se redujeron a 1.8 billones de dólares, equivalentes a sólo un mes del total de importaciones (Lustig, 1998: 25-26).

A partir de entonces y hasta la fecha se han implementado, en todos los niveles, diferentes políticas de modernización económica y de reconversión o reestructuración industrial, acordes al nuevo modelo de desarrollo basado en la exportación de productos manufacturados y no sólo de petróleo. Este proceso de apertura al exterior y de adecuación de la planta industrial mexicana a la competencia internacional, tuvo un momento culminante con la firma del TLCAN. La integración en este bloque económico ha consolidado la dependencia comercial de México con Estados Unidos: en 1993 del total de exportaciones, $82.67 \%$ se realizaban hacia dicho país, mientras que en 2009 la cifra alcanzó 80.51\% (sE, 2010).

La política industrial federal, a principios de los años ochenta, se caracterizó por la reducción de programas y se centró en áreas que se consideraron prioritarias. Así se establecieron tres programas en las siguientes áreas: automotriz, farmacéutica, petroquímica y bienes de capital. Sin embargo, estos programas aún se encontraban, bajo la lógica de la sustitución de importaciones e incluían medidas proteccionistas, como barre- 
ras no tarifarias, importaciones libres de impuestos; y las industrias de autopartes y farmacéutica estaban protegidas por reglas de contenido nacional (Lustig, 1998: 123-124).

Sin embargo esta orientación cambiaría radicalmente en 1985, con el desmantelamiento de los programas industriales, y con la liberalización comercial y la desregulación convirtiéndose en parte integral de la política económica.

En consonancia con el ámbito federal, en el nivel local de Querétaro las políticas de fomento industrial se orientaron sólo hacia políticas fiscales de incentivos (como la exención del impuesto de nómina) con el propósito de atraer inversión foránea, y a la construcción de infraestructura con el propósito de fomentar la inversión y la producción para la exportación, principalmente en lo referente a vialidades y equipamiento urbano. La construcción de nuevos parques industriales quedaría a cargo de la iniciativa privada.

En este sentido, el espacio local de política industrial ha quedado supeditado al ámbito federal, y más aún considerando que su principal rama industrial es la de autopartes, ésta se rige por una regulación supranacional, como es el TLCAN, a partir del cual la protección arancelaria se redujo de $9.9 \%$ en 1994 a cero en 2004; el contenido nacional obligatorio bajó de $34 \%$ a cero para vehículos, y de $20 \%$ a cero para autopartes. La compensación de divisas por empresa se redujo de $80 \%$ a cero; y el nuevo contenido, ahora regional, se elevó de 50 a 62.5\% (Mortimore y Barron, 2005).

En Querétaro, como se puede ver más adelante con la presentación de las estadísticas industriales, la crisis, o más bien la sucesión de crisis, no llevaron a la desaparición de la industria. Por el contrario, a partir de 1976 aumenta el ritmo de implantación de las industrias; entre 1976 y 1989 se instaló 85.3\% de los establecimientos que existían hasta 1990, a pesar de que en este periodo se vivió una de las crisis económicas más profundas. Estos establecimientos industriales pertenecen a diversas ramas productivas que van desde la tradicionales, como las de alimentos y bebidas, hasta las más modernas, como la metal-mecánica, la eléctrica y electrónica (González y Martner, 1990: 25-26). Entre las empresas más importantes que se instalan en este periodo se encuentran: Turborreactores (de inversión pública), Acerlán, Melco y Kimberly Clark (CIEN, 1992: 62).

\section{La estructura industrial de Querétaro}

La importancia del proceso de industrialización desarrollado a partir de los años setenta se puede constatar por los cambios ocurridos en la estruc- 
tura del producto interno bruto (РІв) estatal y en su participación en el ámbito nacional.

Entre 1993 y 2004 la participación de Querétaro en el PIB nacional, en términos reales, aumentó sistemáticamente de 1.40 a $1.76 \%$, alcanzando $1.90 \%$ en 2008 ; crecimiento impulsado por el desarrollo industrial y terciario. De igual manera, la industria manufacturera local elevó su peso en el PIB manufacturero nacional de $2.09 \%$ en 1993 a $2.50 \%$ en 2004, y a $2.54 \%$ en 2008 .

En el ámbito estatal, en 1970 el sector agropecuario representó 17.95\% del PIB, mientras que el sector industrial 36.87\%, y el terciario $45.18 \%$. Casi tres décadas después, en 1999, la estructura se había modificado radicalmente, en detrimento de las actividades primarias que han atravesado por una crisis casi permanente y enfrentan una severa descapitalización, mismas que sólo representaron $2.59 \%$; el sector secundario aumentó a $41.50 \%$, y el avance del terciario se situó en $55.91 \%$. Esta misma tendencia se observa en 2004 con $3.38 \%$ en el sector agropecuario, la minería con sólo $0.69 \%$; la industria $36.54 \%$, continuando el avance del sector terciario hasta alcanzar 59.39\%. En 2008 el rubro agropecuario representó $2.75 \%$, la actividad minera $0.82 \%$, la industria $35.44 \%$, aumentando las actividades terciarias a 60.99 por ciento.

Dada la importancia de la industria manufacturera, se analiza su composición por subsectores, lo cual permite en una aproximación estadística identificar las tendencias de reestructuración, es decir, los subsectores que aumentan su participación en la industria manufacturera, los que entran en crisis y los emergentes.

En 1993 el universo de la industria manufacturera en Querétaro estaba constituido por 3,054 establecimientos que dieron empleo a un total de 60,518 trabajadores. Entre los diferentes subsectores destacó en primer lugar el metal-mecánico, donde se concentraron 852 establecimientos $(27.90 \%$ ) y dio empleo a 23,668 trabajadores que representaron $39.11 \%$ del personal ocupado en la manufactura, y generó $38.27 \%$ del valor agregado bruto. En especial destaca la fabricación de partes para el sistema de transmisión de automóviles, la fabricación de aparatos de uso doméstico y la de maquinaria y equipo eléctrico (cuadro 1).

Las industrias tradicionales, como la de alimentos y la textil, con un asentamiento más antiguo en Querétaro, se encontraban en segundo y tercer lugar, respectivamente. La industria de alimentos representó $27.47 \%$ de los establecimientos, dio empleo a $17.25 \%$ de los trabajadores de la manufactura y generó $23.77 \%$ del valor agregado bruto. La industria textil contaba con $13.10 \%$ de los establecimientos y empleó a $16.55 \%$ del personal ocupado, sin embargo, sólo participó con $6.82 \%$ del valor agregado bruto. 


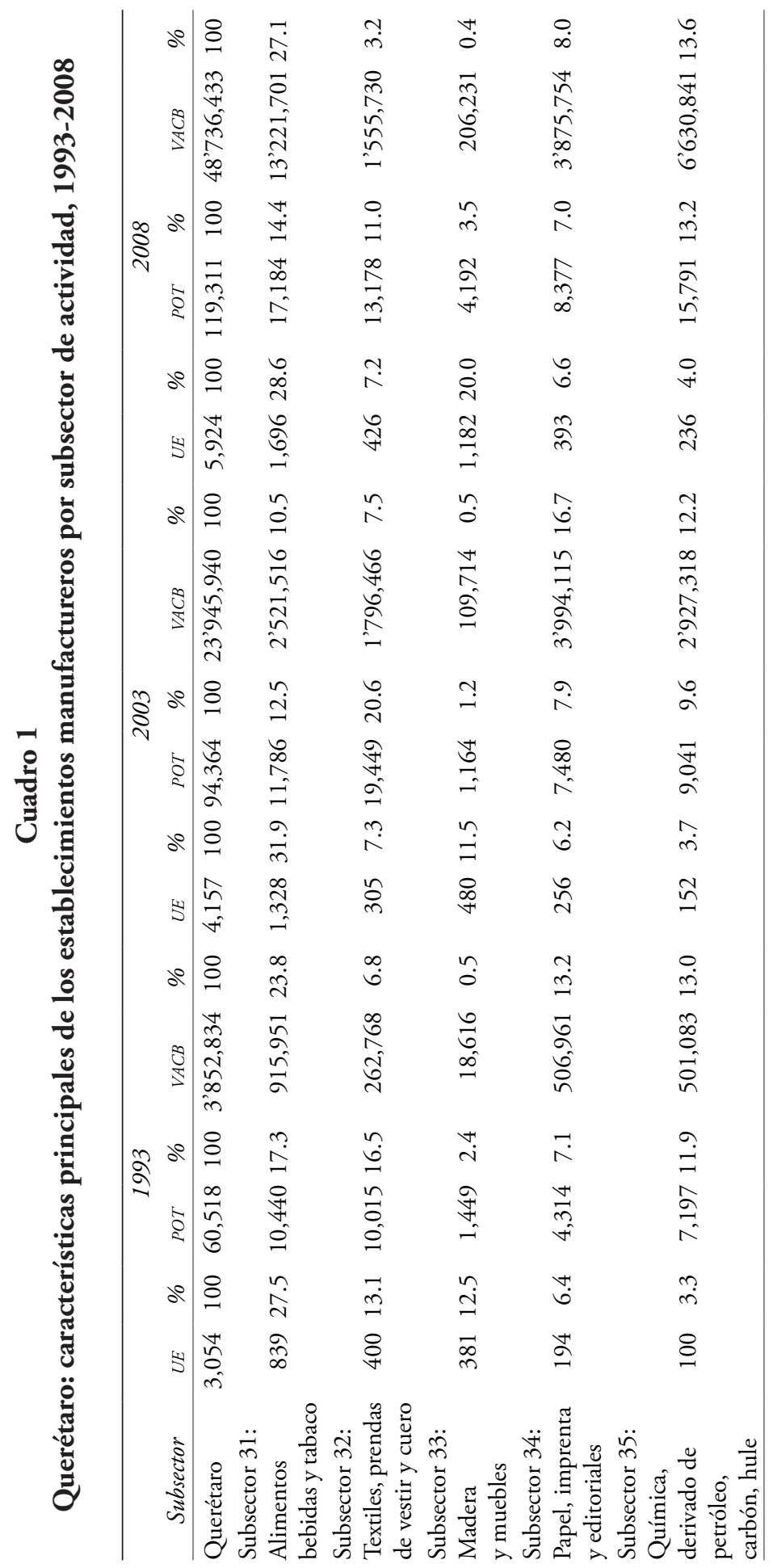




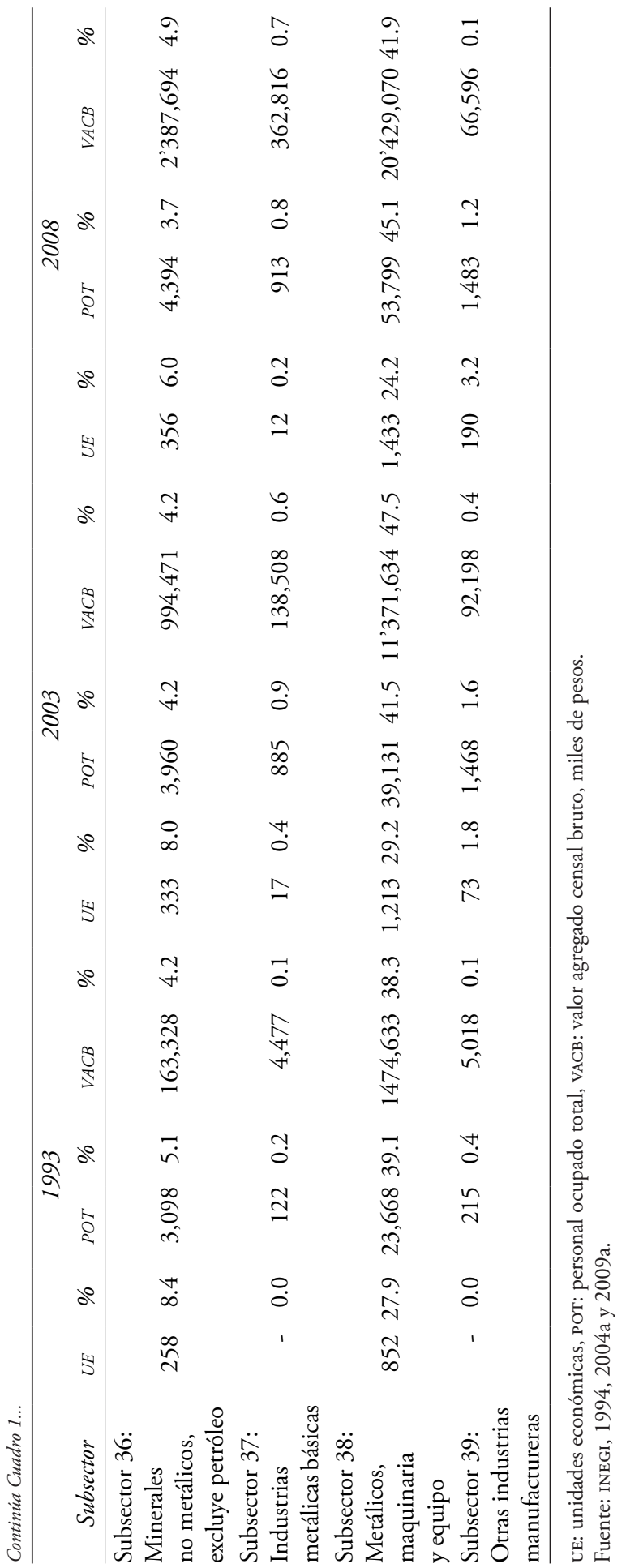


Aunque no son tan importantes por el número de establecimientos o por el empleo, los sectores del papel y de la química tuvieron relevancia en cuanto a la generación de valor agregado, contribuyendo con 13.16 y $13.01 \%$, respectivamente.

En 2003 existían ya un total de 4,157 establecimientos manufactureros, con un personal ocupado total de 94,364. En los subsectores mantiene su primacía el metal-mecánico, con $47.5 \%$ del valor agregado industrial, con $41.5 \%$ del empleo y concentra $29.2 \%$ de los establecimientos. La industria textil y la de alimentos mantienen su importancia por el número de trabajadores: representa 20.6 y $12.5 \%$; a 7.3 y $31.9 \%$ de los establecimientos y generan 7.5 y $10.5 \%$ del valor agregado, respectivamente. Al igual que en 1993, la industria del papel es importante por el valor agregado generado, el cual alcanzó $16.7 \%$; con $9.6 \%$ del total de trabajadores y $6.2 \%$ de los establecimientos. La industria química representó $12.2 \%$ del valor agregado, dando empleo a $9.6 \%$ de los trabajadores, con sólo 3.7\% de los establecimientos.

En 2008 el número de establecimientos aumentó a 5,924 y el de trabajadores a 119,311. Nuevamente con el primer lugar se situó el subsector de maquinaria y equipo con $41.9 \%$ del valor agregado, y con $45.1 \%$ de los trabajadores. En el segundo puesto se situó la industria alimentaria con $27.1 \%$ del valor agregado y $14.4 \%$ del empleo.

$\mathrm{Al}$ interior de la industria manufacturera destacó la industria automotriz con $19.6 \%$ del personal ocupado y $20.2 \%$ del valor agregado.

\section{Concentración geográfica de la industria}

Las políticas de desconcentración de actividades de la ciudad de México, que impulsaron el desarrollo industrial de Querétaro a partir de los años setenta, se tradujeron en un proceso de descentralización pero en la misma región centro, la más industrializada del país, al interior de la cual la ciudad de Querétaro es la más alejada: se encuentra a $211 \mathrm{~km}$ de la ciudad de México (Garza, 1992).

En cuanto al territorio, el crecimiento industrial en el estado de Querétaro ha tendido a reproducir paradójicamente, y en su escala, el fenómeno de concentración que se da en el ámbito nacional, ya que desde los inicios del proceso de industrialización los parques y las zonas industriales se desarrollaron sobre todo en la periferia de las ciudades históricamente más importantes: Querétaro y San Juan del Río, y después en otros municipios. Esta creación de asentamientos industriales y el trazo de la autopista han determinado sustancialmente el patrón de localización de los establecimientos industriales. De igual manera, influye el periodo en 
que iniciaron operaciones las empresas, pues las más antiguas se ubicaron en los espacios industriales que se crearon desde los años cuarenta.

En la actualidad la entidad cuenta con 20 parques industriales en operación, de los cuales seis se ubican en el municipio de Querétaro, siete en El Marqués, tres en San Juan del Río, dos en Colón y dos en Corregidora (GEQ, 2009: 164-166).

En consecuencia se puede observar una alta concentración de la actividad manufacturera en dos polos, fuera de los cuales la actividad industrial es poco significativa. El primero y más importante dio origen a la Zona Metropolitana de Querétaro (ZMQ), conformada por los municipios de Querétaro, El Marqués, Corregidora y Huimilpan, y el segundo por San Juan del Río y Tequisquiapan, conformando el corredor industrial Querétaro-San Juan del Río.

En 1993 la concentración del número total de establecimientos en la ZMQ alcanzó $57.10 \%$, mientras que en San Juan del Río y Tequisquiapan el $25.44 \%$. En la zMQ el personal ocupado fue de $62.84 \%$, y en el otro polo de $29.08 \%$. La generación de valor agregado fue de $69.04 \%$ y $25.36 \%$, respectivamente.

La tendencia registrada en 2003 muestra casi la misma concentración espacial. La ZMQ redujo su concentración a 54.91\% de los establecimientos, mientras que San Juan del Río y Tequisquiapan aumentaron a 30.50\%. Si se toma en cuenta el personal ocupado, la ZMQ redujo ligeramente su peso y representó $61.62 \%$. Por su parte, San Juan del Río y Tequisquiapan aumentaron su importancia a $31.22 \%$. Sin embargo, si se considera el valor agregado, se observa que la ZMQ aumentó significativamente su participación a 75.54\%; lo cual permite hablar de una mayor productividad y eficiencia; mientras que la otra concentración industrial disminuyó su contribución a 20.9\% (cuadro 2).

En 2008, considerando el valor agregado, la ZMQ continuó siendo la más relevante, aunque redujo ligeramente su participación a $73.7 \%$; el personal ocupado aumentó de manera significativa a 69.9\%; mientras que su participación en los establecimientos (54.7\%) se mantuvo prácticamente igual. La concentración industrial de San Juan del Río y Tequisquiapan, en relación con el valor agregado, aumentó un poco su aportación a $22.2 \%$; mientras que tuvieron una baja importante en el personal ocupado con $23.1 \%$; el peso en los establecimientos se mantuvo prácticamente igual, con 31.5 por ciento.

Por su parte, en 1993 la industria automotriz en el ámbito estatal estaba constituida por 38 establecimientos, que ocuparon a un total de 5,722 trabajadores. Ya desde este año mostraba una alta concentración en el municipio de Querétaro: $60.5 \%$ de los establecimientos, sin que algún otro municipio representara al menos 1\%, 72.9\% de los trabaja- 


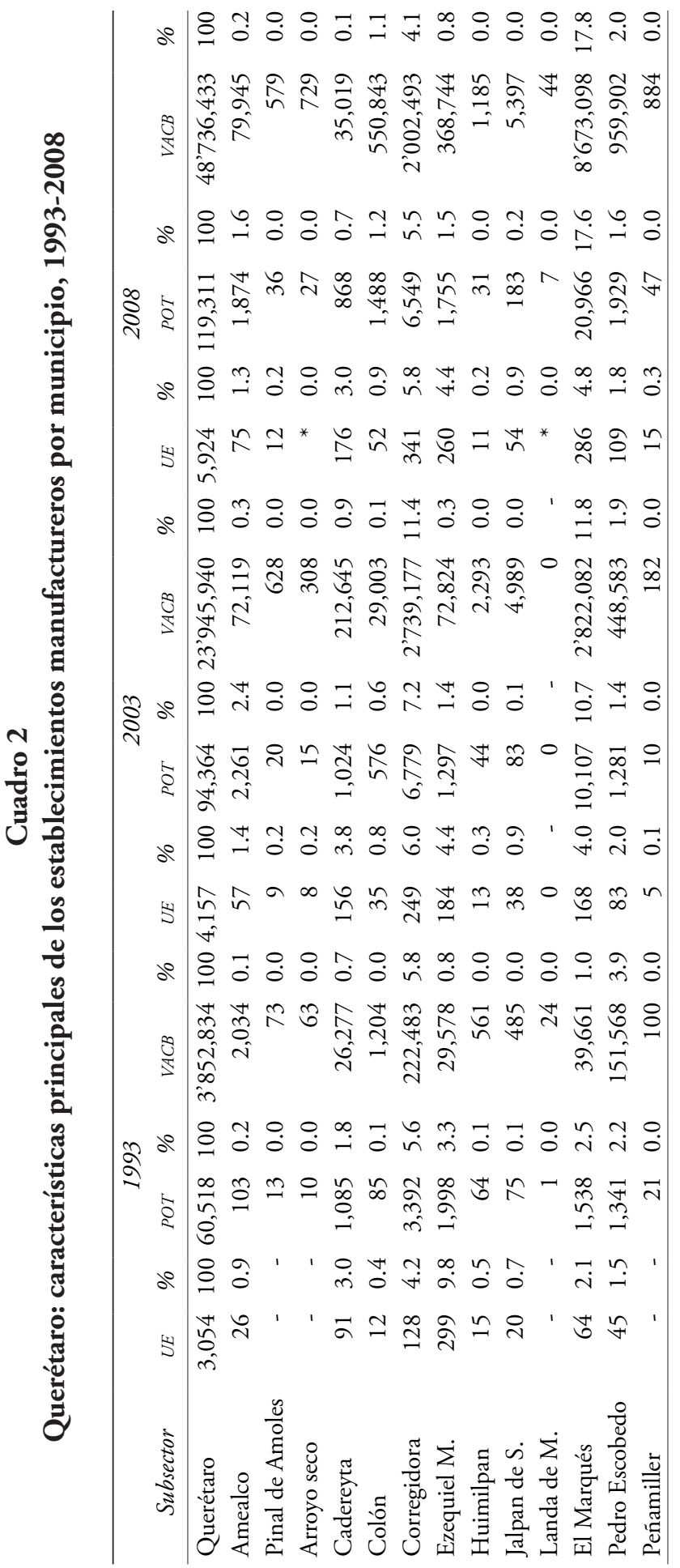




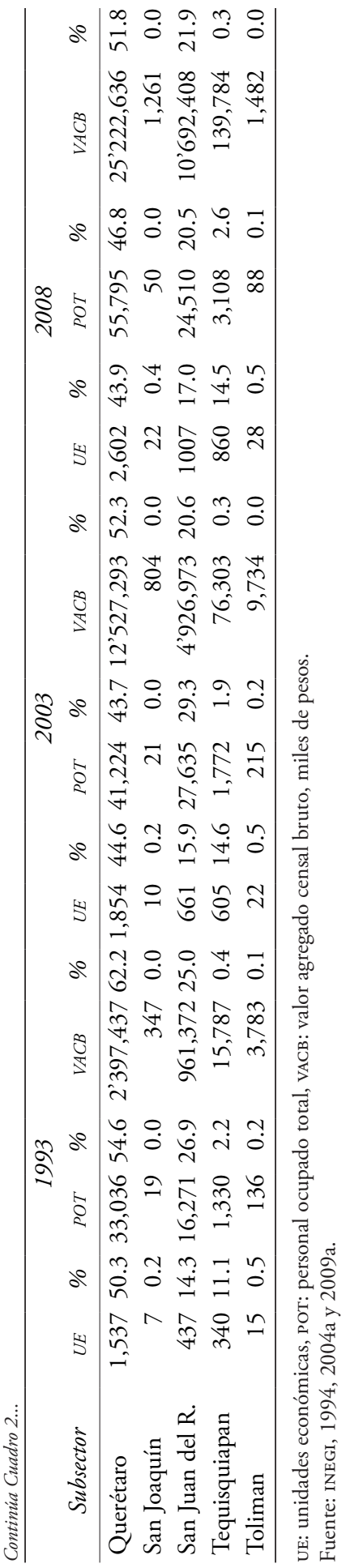


dores y $72.2 \%$ del valor agregado; en segundo lugar se encontraba el municipio de Pedro Escobedo con $22.7 \%$ del valor agregado. Por su parte, San Juan del Río concentró $11.4 \%$ del personal ocupado y sólo $4.5 \%$ del valor agregado.

En 2003 ya se puede observar claramente la tendencia a que la industria de autopartes se localice, además de en el municipio de Querétaro, en otros dos municipios de la zona metropolitana que antes no eran significativos. El predominio del municipio de Querétaro en esta actividad continuó en términos del número de empresas, pues su participación aumentó ligeramente a $61.2 \%$, aunque disminuyó si se consideran otras variables. Su participación en el número de trabajadores descendió a $58.1 \%$, mientras que el valor generado lo hizo a $69.5 \%$. El Marqués fue el municipio que mostró el mayor aumento de importancia, dado que concentró a $16.4 \%$ de los establecimientos, $25.2 \%$ del empleo de la rama y $23.4 \%$ de la generación del valor agregado. El otro municipio relevante fue Corregidora, que representó $12.2 \%$ de los trabajadores y $4.7 \%$ del valor agregado. En contraste, San Juan del Río contribuyó con $10.4 \%$ de los establecimientos, pero sólo $1.9 \%$ de los trabajadores y $0.7 \%$ del valor agregado (cuadro 3).

En 2008 se acentuó la alta concentración de la actividad automotriz en sólo dos municipios. Querétaro representó 55\% del valor agregado mientras que El Marqués $41.8 \%$, totalizando ambos $96.8 \%$. En términos del número de trabajadores las aportaciones fueron de 62.2 y $32.8 \%$, respectivamente, alcanzando en conjunto $95 \%$, con un total de 22,217 trabajadores.

\section{Evolución de la industria de autopartes en Querétaro}

La industria de autopartes en Querétaro, como se mencionó, tuvo sus inicios en los años sesenta como parte de la estrategia de sustitución de importaciones de maquinaria y equipo, pero con inversiones que provinieron de fuera del estado tanto nacionales como extranjeras, donde destacan las inversiones realizadas por el grupo Spicer y Tremec. Esta actividad manufacturera inició como la implantación de un giro totalmente nuevo en la entidad que no buscaba potenciar una tradición en actividades de forja y fundación pues no existía, ni aprovechar las actividades siderúrgicas, y que tampoco trataba de integrar una materia prima como sería el hierro, dado que no existen yacimientos en la entidad.

Desde fines de los años noventa, la industria automotriz de Querétaro ha cobrado importancia en el ámbito nacional al pasar del décimo lugar en 1993 con $2.9 \%$ del valor agregado total de la industria, al octavo en 1998 con 4.9\%; lugar que mantuvo en 2003 con la misma parti- 


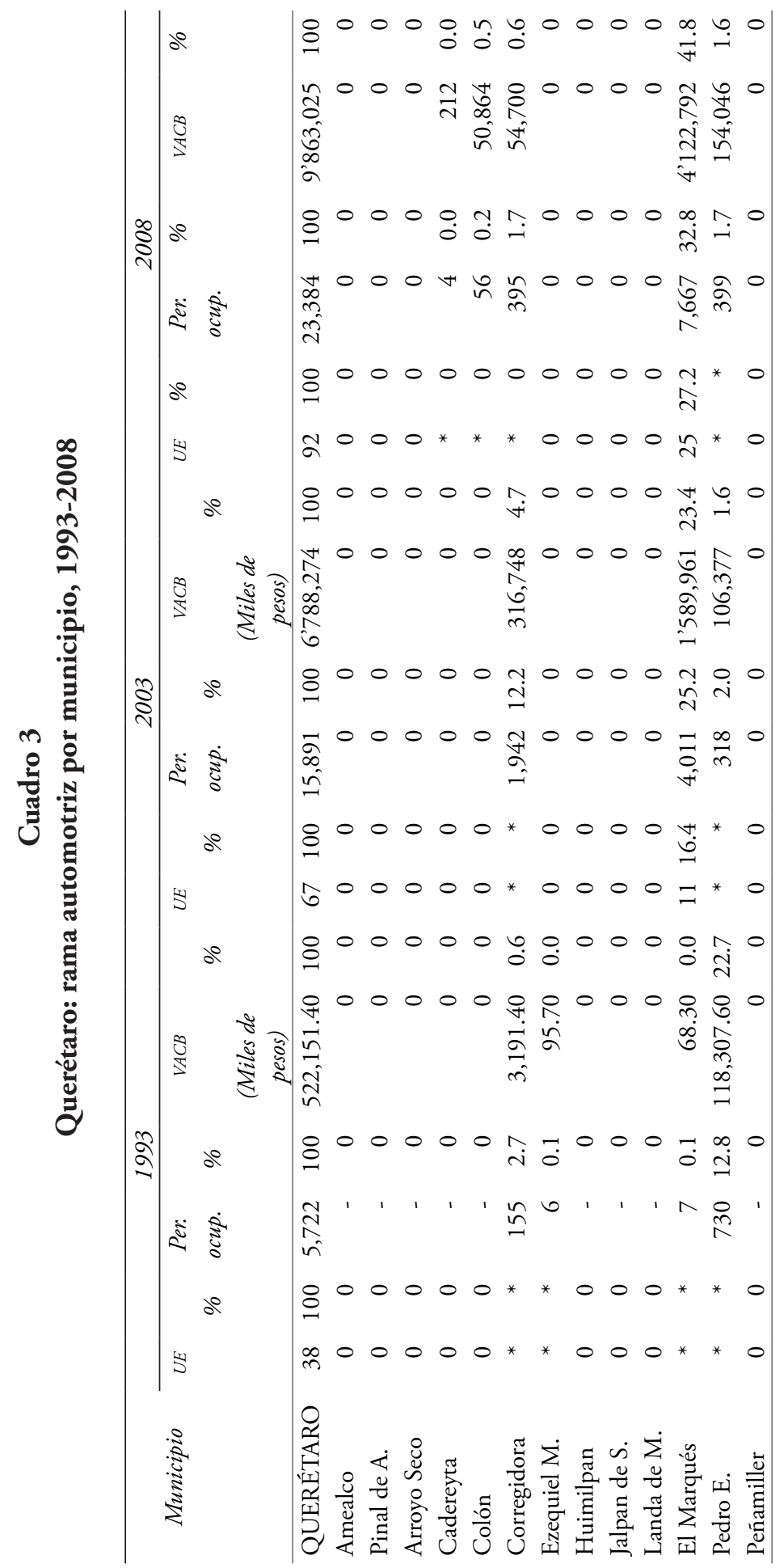




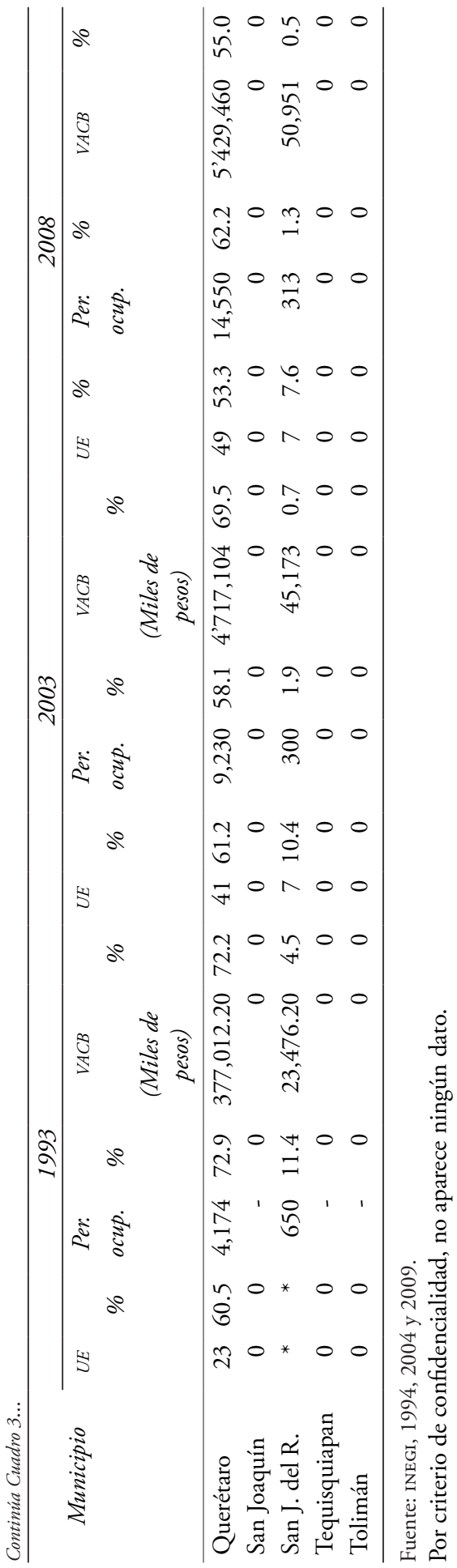


cipación de 4.9\%. En 2008 Querétaro se situó en el noveno sitio y registró casi la misma participación: 4.6\% (INEGI, 2002, 2006, 2010). El lugar que ha ocupado Querétaro en el concierto internacional es aún más significativo si se considera que los estados que lo anteceden tienen plantas ensambladoras o de motores. La industria automotriz de Querétaro, como mencionamos, se ubica en el rubro de autopartes.

En 1993, en la industria de autopartes de Querétaro y según el valor agregado generado, la actividad más importante fue la producción de transmisiones y sus partes, con $61.5 \%$ del total, seguida de la manufactura de equipo eléctrico y electrónico con $18.7 \%$, en tercer lugar se encontraba la fabricación de otras partes y accesorios con 13.4\%, mientras que en el cuarto se ubicó la actividad de motores y sus partes con 4.1 por ciento.

En 2003 un aspecto de la evolución de la rama de autopartes se manifestaba en la redistribución ocurrida en términos de las diferentes actividades. Considerando igualmente el valor agregado, se pudo observar que el rubro de otras partes registró el mayor aumento en términos de su importancia local: alcanzó $41.9 \%$ con el primer lugar, seguido por la producción de transmisiones con $22.5 \%$, mientras que en tercer sitio se encontraba la manufactura de equipo eléctrico y electrónico, con $14.9 \%$. Las otras actividades en orden de importancia fueron: los sistemas de dirección y suspensión con $12.9 \%$ y la de frenos y sus partes con el $6.9 \%$ (cuadro 4).

En 2008 se acentuó la importancia de la producción de otras partes, alcanzó 44.2\%; e igualmente en segundo lugar se ubicó la actividad de transmisiones con $21.6 \%$, y en tercero la de equipo eléctrico y electrónico con 14.4 por ciento.

El dinamismo de esta rama se puede explicar por el monto de inversión extranjera, el cual en 2009 representó $25.6 \%$ del total de la efectuada en el estado de Querétaro (SE, 2010). Si se observa la balanza comercial de Querétaro se tiene que, en 2009, las exportaciones alcanzaron 1,366 millones de dólares, mientras que las importaciones 1,558.6 millones de dólares, con un saldo en contra de 192.6 millones de dólares (GEQ, 2010).

Durante la crisis mundial de la industria automotriz ocurrida a finales de 2008 y 2009, en Querétaro la mayoría de las empresas del sector implementaron medidas como paros técnicos y recortes de personal; y el cierre de operaciones de las siguientes empresas: American Car Equipment, Arneses Eléctricos Automotrices, Arvin Meritor, y Automotive Lighting. $\mathrm{Al}$ igual que en el ámbito nacional, el sector muestra signos relativos de recuperación, pues mientras en diciembre de 2009 trabajaban 27,122 empleados, en junio de 2010 aumentaron a 30,736 (GEQ, 2010). 


\section{Cuadro 4 \\ Querétaro: valor agregado de la industria automotriz por subrama de actividad, 1993-2008 (miles de pesos)}

\begin{tabular}{lrrrrrr}
\hline & \multicolumn{2}{c}{1993} & \multicolumn{2}{c}{2003} & \multicolumn{2}{c}{2008} \\
\hline Total & $642,213.1$ & 100.0 & $6 ’ 788,274$ & 100.0 & $9 ’ 863,025$ & 100.0 \\
Ensamble de autos & $2,755.9$ & 0.4 & 0 & - & 254,590 & 2.6 \\
y camiones & & & & & & \\
Carrocerías & $3,679.1$ & 0.6 & 29,111 & 0.4 & 49,354 & 0.5 \\
Motores & $26,164.9$ & 4.1 & 32,418 & 0.5 & 219,421 & 2.2 \\
Equipo eléctrico & $120,061.7$ & 18.7 & $1^{\prime} 011,089$ & 14.9 & $1,422,547$ & 14.4 \\
y electrónica & & & & & & \\
Sistema de dirección & 356.2 & 0.1 & 873,252 & 12.9 & 192,739 & 2.0 \\
y suspensión & & & & & & \\
Frenos & $8,293.7$ & 1.3 & 471,263 & 6.9 & 402,709 & 4.1 \\
Transmisiones & $395,043.5$ & 61.5 & $1^{\prime} 524,212$ & 22.5 & $2 ' 130,633$ & 21.6 \\
Asientos & - & - & 278,903 & 4.1 & 538,467 & 5.5 \\
Troquelados & - & - & 22,991 & 0.3 & 288,276 & 2.9 \\
Otras partes & $85,858.1$ & 13.4 & $2 ’ 545,035$ & 37.5 & $4 ’ 364,289$ & 44.2 \\
\hline
\end{tabular}

Nota: En 1993 los rubros de asientos y piezas troqueladas estaban comprendidos en otras partes. Fuente: INEGI, 1994, 2004b, 2009 b.

\section{Las empresas de autopartes}

El universo de empresas de autopartes incluido en este estudio se conformó por 127 empresas, de las cuales ocho son grandes, 42 medianas, 35 pequeñas y 42 micro.

De estas empresas, 47 eran proveedores de primer nivel (TR1) y representaron $13 \%$ de las empresas de esta categoría en el ámbito nacional, ubicando a Querétaro en el primer lugar, donde el estado de Coahuila tuvo 46, Chihuahua 43 y Monterrey 35 (GEQ, 2004). Entre ellas se pueden mencionar: Forjas Spicer, Arvin de México, Meritor Mexicana, American Car Equipment, Tremec, Valeo Silvana Iluminación, Industrias Michelin, Johnson Matthey de México, TRw Inc., Ventramex y Magnoflex.

La mayor parte de estas empresas son filiales de grandes consorcios internacionales de autopartes, que desde los años ochenta, y en mayor medida durante los noventa, han seguido a las grandes compañías trasnacionales armadoras en sus relocalizaciones geográficas, orientadas principalmente hacia países de la periferia de industrialización importante, pero con salarios mucho menores y con regulaciones más laxas, entre los que se encuentran los países de Europa del Este, Corea, China, Brasil o México.

Esta reestructuración industrial y territorial ha tenido como una de sus consecuencias principales una selección y concentración de produc- 
tores que ha dejado fuera de la proveeduría directa a miles de empresas pequeñas y micro que no pueden tener acceso al know how de producción, ni a la innovación de procesos y productos exigidas por las armadoras en el marco del nuevo modelo productivo (Juárez, 2005: 78-83).

Para profundizar el análisis de la industria de autopartes en Querétaro se presentan a continuación los resultados de la información proveniente de un cuestionario aplicado en 13 empresas de la rama, las cuales fueron seleccionadas mediante un muestreo aleatorio estratificado.

\subsection{Localización de las empresas}

Como se señaló, el estado de Querétaro cuenta con 20 parques industriales, en los cuales se localiza la mayoría (11) de las empresas encuestadas, sin que exista una marcada tendencia a localizarse en uno específico. Por ejemplo, cuatro se ubican en el Parque Industrial Bernardo Quintana, del municipio El Marqués, se trata de empresas grandes, medianas y pequeñas; dos se localizan en el Parque Industrial Benito Juárez, municipio de Querétaro (una grande y una pequeña), mientras que el resto se ubica cada una en un espacio industrial distinto. La localización de las empresas por municipio refleja la concentración existente tanto de la industria manufacturera en general, como la de autopartes en lo particular, al ubicarse 10 empresas en los dos municipios más importantes, cinco en Querétaro y cinco en El Marqués (cuadro 5a).

\section{Cuadro 5a}

\section{Localización y tamaño}

\begin{tabular}{clll}
\hline Empresa & Tamaño & \multicolumn{1}{c}{ Localización } & \multicolumn{1}{c}{ Municipio } \\
\hline 1 & Grande & Zona Industrial & San Juan del Río \\
2 & Grande & P.I.B. Quintana & El Marqués \\
3 & Grande & Frac. I. Balvanera & Corregidora \\
4 & Grande & P.I.B. Juárez & Querétaro \\
5 & Mediana & P.I. El Tepeyac & El Marqués \\
6 & Mediana & P.I.B. Quintana & El Marqués \\
7 & Mediana & P.I.B. Quintana & El Marqués \\
8 & Pequeña & P.I.B. Quintana & El Marqués \\
9 & Pequeña & Zona Industrial & San Juan del Río \\
10 & Pequeña & P.I.B. Juárez & Querétaro \\
11 & Micro & Zona Industrial Jurica & Querétaro \\
12 & Micro & Frac. I. Carrillo Puerto & Querétaro \\
13 & Micro & Centro Histórico & Querétaro \\
\hline
\end{tabular}

Fuente: Información derivada de las encuestas del trabajo de campo. 


\subsection{Tamaño de los establecimientos y origen del capital}

En la determinación del tamaño de las empresas se consideró sólo el número de empleados. ${ }^{1}$ De acuerdo con el muestreo utilizado, de las 13 empresas que respondieron el cuestionario, cuatro son grandes, tres medianas; tres pequeñas y tres micro.

Como podría esperarse, entre las empresas se encontró el predominio del carácter de sucursales de grandes consorcios trasnacionales de autopartes con nueve empresas, y las cuatro restantes son establecimientos únicos. De las empresas que son sucursales, salvo en un caso, todas tienen inversión extranjera al $100 \%$, predominando ligeramente la proveniente de Estados Unidos.

Esta composición refleja las características de los modelos de industrialización seguidos en México, principalmente en la industria metalmecánica a la que pertenece la rama de autopartes que se ha desarrollado a partir de la inversión extranjera, autorizada en la actualidad hasta el $100 \%$. Y más recientemente, a partir del proceso de reorientación de la economía y de la producción industrial hacia el exterior, así como de la conformación de grandes bloques económicos regionales, las características de las empresas estudiadas reflejan el papel de México como una plataforma de exportación, pues si se toma en cuenta el año de inicio de las operaciones, se observó que 10 de las 13 empresas arribaron a Querétaro durante el periodo comprendido de fines de los años ochentas a 2001, en respuesta a la apertura económica hacia el exterior (cuadro 5b).

\subsection{Relación entre las empresas y su casa matriz}

Considerando que un aspecto importante de este trabajo de investigación está constituido por la indagación acerca del papel de las empresas de autopartes de Querétaro en las cadenas productivas, se investigó acerca de los vínculos existentes entre las sucursales y sus casas matrices. Sobre las decisiones tomadas por la casa matriz, se encontró que mantienen el control en las actividades más importantes que requieren de investigación y desarrollo, como el diseño del producto, así como el establecimiento de acuerdos comerciales y la introducción de innovaciones tecnológicas (cuadro 5c).

\footnotetext{
${ }^{1}$ Se utilizó la clasificación proveniente del Directorio Maestro Empresarial, 2002, de acuerdo con el cual la empresa grande ocupa a más de 501 trabajadores; la mediana de 101 hasta 500; la pequeńa de 31 a 100; mientras que la micro tiene de 1 a 30 empleados, dado que la muestra se determinó a partir de esta fuente.
} 


\section{Cuadro 5b}

Tamańo de empresa y origen del capital

\begin{tabular}{cllcclcc}
\hline Empresa & Tamaño & $\begin{array}{c}\text { Pertenencia a } \\
\text { consorcio }\end{array}$ & IED & $\%$ & \multicolumn{1}{c}{ País } & $\begin{array}{c}\text { Inicio de } \\
\text { operaciones }\end{array}$ \\
\hline 1 & Grande & Clarion & Sí & 100 & Japón & 1989 \\
2 & Grande & Nihon Plast & Sí & 100 & Japón & 1995 \\
3 & Grande & Prettl & Sí & 100 & Estados Unidos & 1996 \\
4 & Grande & Desc & Sí & 49 & Estados Unidos & 1971 \\
5 & Mediana & Arvin Meritor & Sí & 100 & Estados Unidos & 2001 \\
6 & Mediana & Woco & Sí & 100 & Canadá & 1994 \\
7 & Mediana & Johnson Matthey & Sí & 100 & Inglaterra & 1995 \\
8 & Pequeña & Shimizu Kogyo & Sí & 100 & Japón & 1998 \\
9 & Pequeña & Ninguno & No & 100 & México & 1998 \\
10 & Pequeña & Peterson American & Sí & 100 & Estados Unidos & 1982 \\
11 & Micro & Ninguno & No & 100 & México & 1989 \\
12 & Micro & Ninguno & No & 100 & México & 1998 \\
13 & Micro & Ninguno & No & 100 & México & 1947 \\
\hline
\end{tabular}

Fuente: Información derivada de las encuestas del trabajo de campo.

\section{Cuadro 5c \\ Relación empresas y casa matriz}

\begin{tabular}{llllll}
\hline Empresa & Tamaño & $\begin{array}{c}\text { Pertenencia a } \\
\text { consorcio }\end{array}$ & $\begin{array}{c}\text { Tipo de } \\
\text { integración }\end{array}$ & $\begin{array}{c}\text { Tipode } \\
\text { producto }\end{array}$ & $\begin{array}{c}\text { Decisiones } \\
\text { tomadas por la } \\
\text { casa matriz }\end{array}$ \\
\hline 1 & Grande & Clarion & Mixta & Final & $\begin{array}{l}\text { DP, AC, EV, IT } \\
\text { DP, AC, EV, IT }\end{array}$ \\
2 & Grande & Nihon Plast & Ninguna & & Ninguna \\
3 & Grande & Prettl & Ninguna & Ninguna & Ninguna \\
5 & Grande & Desc & Mixta & Final & DP, AC, IT \\
6 & Mediana & Arvin Meritor & Horizontal & Final & DP, GP, AC, EV, IT \\
7 & Mediana & Woco & Final & DP, AC, IT \\
8 & Mediana & Johnson Matthey & Mixta & AC \\
9 & Pequeña & Shimizu Kogyo & Ninguna & & \\
10 & Pequeña & Peterson American & Ninguna & Final & DP, AC, EV, IT \\
11 & Micro & Ninguno & Ninguna & & \\
12 & Micro & Ninguno & Ninguna & & \\
13 & Micro & Ninguno & Ninguna & & \\
\hline
\end{tabular}

Nota: DP, diseño del producto; GP, gestión del proceso; CP, contratación de personal; AC, acuerdos comerciales; EV, estrategia de ventas; IT, innovaciones tecnológicas.

Fuente: Información derivada de las encuestas del trabajo de campo. 


\subsection{Producción y ventas}

Las empresas de autopartes encuestadas se dedicaban a la fabricación de diferentes productos, entre los que se encuentran equipo de audio automotriz, volantes, arneses, flechas cardán, elevadores para ventanas, soportes para motor, convertidores catalíticos, troquelado de metales, soportes para ejes, resortes de válvulas de motor, procesos de zincado electrolítico, herramientas para ensamble y electrodos.

La mayor parte de las empresas realizan sus ventas tanto en el mercado nacional como en el extranjero, siendo Estados Unidos el principal país de destino de las exportaciones. De esta manera México se confirma como una atractiva y competitiva plataforma para la exportación hacia el importante mercado estadounidense; que en el caso de Querétaro, en la cadena productiva internacional, corresponde a la producción de mercancías tanto de mayor tecnología relativa como sistemas de transmisiones, equipo eléctrico y electrónico, sistemas de dirección y suspensión; como a las de menor tecnología (cuadro 5d).

\subsection{Relaciones establecidas con los clientes}

La relación que las empresas estudiadas establecen con sus clientes varía significativamente de acuerdo con el tamaño de la empresa y el origen del capital. Las empresas grandes y medianas venden el total o un alto porcentaje de sus productos a una armadora automotriz, entre éstas se encuentran Nissan, Volkswagen, Ford o General Motors, lo que las ubica como proveedoras de primer nivel, o tier one, beneficiándose de los contratos establecidos desde su país de origen por su casa matriz, que son grandes compañías trasnacionales de autopartes. En correspondencia, el tipo de contrato que establecen con su clientes es de largo plazo y permanente, y realizan entregas justo a tiempo.

Por su parte, las empresas de menor tamaño, micro y pequeñas se sitúan como empresas de segundo nivel en la cadena automotriz, con contratos de largo plazo y permanentes, pero no realizan entregas justo a tiempo (cuadro 5d).

\subsection{Insumos}

El predominio de los encadenamientos internacionales, así como un mayor alcance del mercado también se pudo observar al considerar las compras de insumos. Este proceso, al igual que al analizar las variables mencionadas, es claro entre las empresas de mayor tamańo, las grandes y las medianas, donde predomina el capital de origen extranjero. Esta ca- 


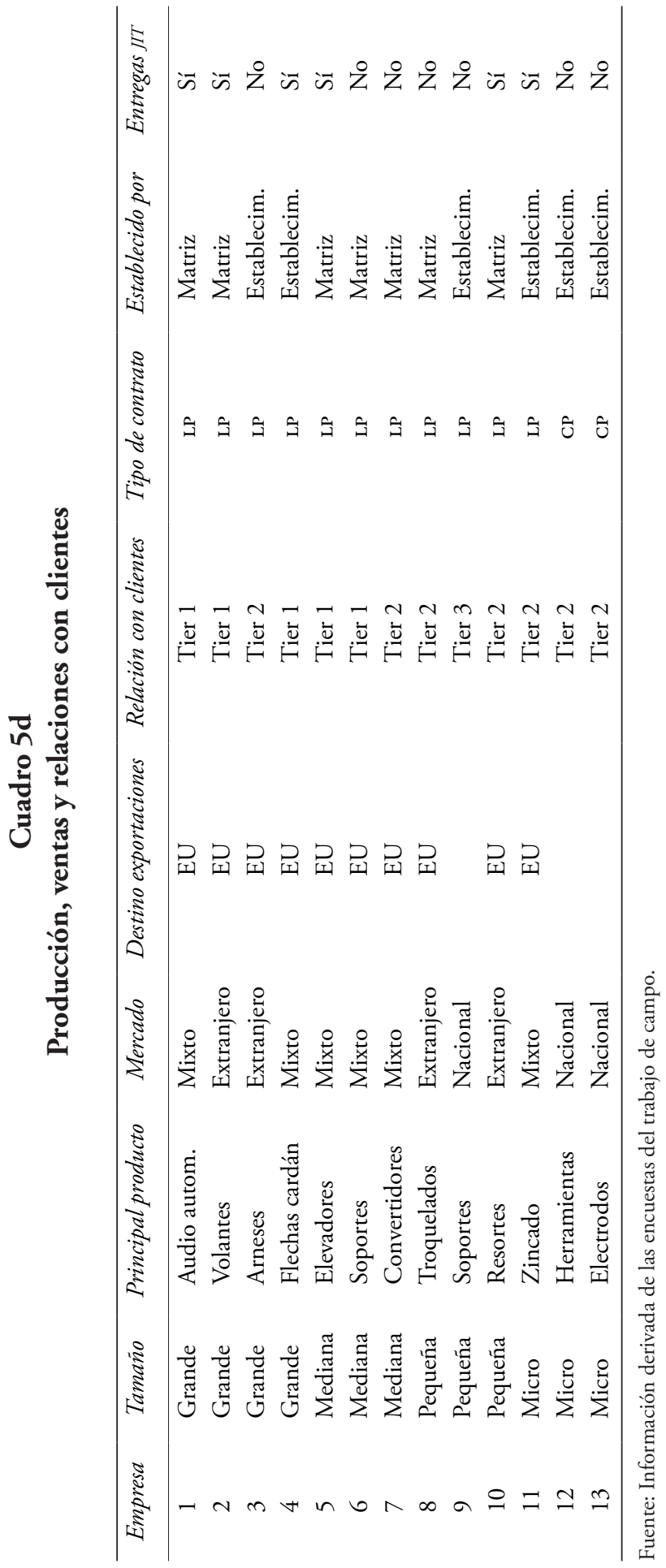


racterística, aunque no sólo relacionada con las compras de insumos, sino también con las ventas de productos realizadas por la industria de autopartes, fue observada por González y Nieto (2007).

Entre los motivos más importantes señalados por las empresas para tener que recurrir a las importaciones de insumos sobresalen el menor costo y la calidad. Entre los insumos importados destacaron: componentes electrónicos, partes metálicas, partes plásticas, cerámica, forjas, fundiciones y lámina de acero (cuadro $5 \mathrm{e}$ ).

El predominio de actividades de ensamble con insumos importados, más que de fabricación en el sentido tradicional, no es privativa de Querétaro, también ha sido observada en Argentina (Motta et al., 2000; Novick y Yoguel, 2001).

De igual manera, la fuerte relación con el ámbito internacional se pudo observar dado que en la totalidad de los casos la maquinaria y el equipo son importados.

\section{Conclusiones}

El proceso de reestructuración industrial en Querétaro, en general, y el de la rama de autopartes en particular, ha tendido a reforzar la concentración de la actividad en municipios que conforman la ZMQ. En especial han cobrado mayor importancia los municipios de El Marqués y Corregidora. Mientras que el municipio de San Juan del Río, el otro polo industrial del estado, ha perdido importancia, y en el caso de la industria de autopartes es casi marginal.

El patrón de localización industrial ha estado fuertemente determinado por la ubicación estratégica de Querétaro, gran cercanía con la ciudad de México y paso casi obligado hacia el norte del país, que se ha visto reforzada por la construcción y ampliación de la autopista México-Querétaro. De igual manera ha tenido una influencia importante la política federal de desconcentración de actividades del Distrito Federal desarrollada en la década de los setenta. Esta misma ubicación le ha permitido al estado de Querétaro adaptarse con mayor éxito relativo a los diferentes modelos productivos que a otras entidades del país, ya que puede seguir teniendo un fácil acceso al principal mercado interno, la Zona Metropolitana de la Ciudad de México, así como hacia el norte del país.

Las empresas de autopartes estudiadas pertenecen prácticamente a dos universos diferentes y reflejan el carácter polarizado y concentrado del proceso de reestructuración de la rama.

Las empresas grandes y medianas se caracterizan por el predominio de la inversión extranjera al 100\%, cuyo origen principal es Estados Unidos, seguido de Japón, Inglaterra y Canadá; estas inversiones en su 


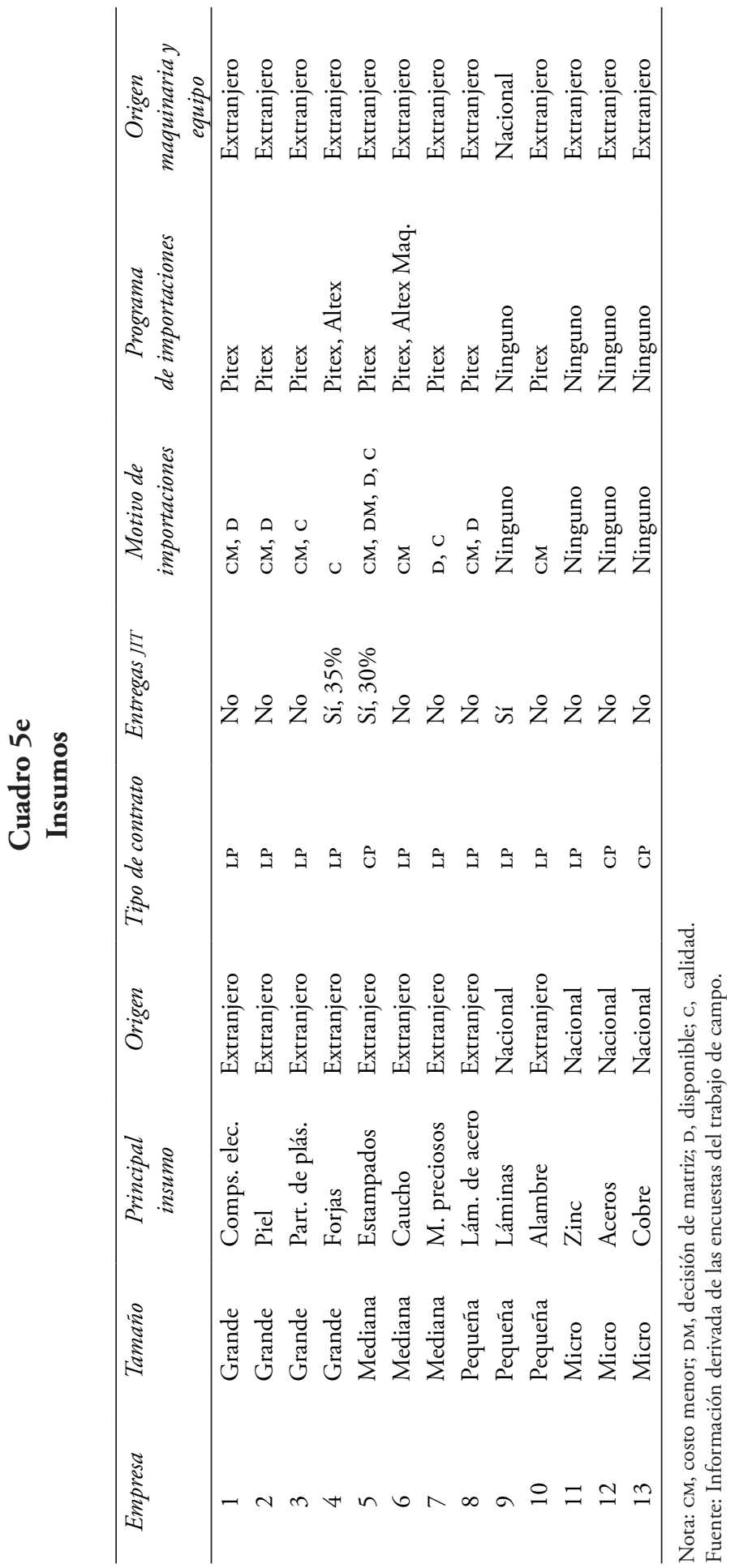


totalidad han sido realizadas por grandes consorcios de autopartes internacionales. Estos grupos responden a la lógica de reestructuración global de la industria, la cual tiende a localizarse fuera de sus lugares de origen pero cerca de mercados importantes.

Estas empresas son las que presentan las mayores ventajas en cuanto a calidad y productividad, lo que les permite orientar principalmente su producción hacia el extranjero ya sea directa o indirectamente. Las ventas que realizan en el mercado nacional se destinan sobre todo a la plantas armadoras, constituyéndose en proveedores directos o de primer nivel, o bien de segundo nivel, con certificados de calidad internacionales.

El otro grupo de empresas, conformado por las pequeñas y micro, presenta características muy diferentes. En primer lugar destaca el origen de la inversión que es predominantemente nacional al 100\%, salvo en dos casos donde la inversión es $100 \%$ extranjera y proviene de Estados Unidos y Japón. Su producción está orientada en lo fundamental al mercado nacional, a excepción de los mismos casos en donde la inversión es extranjera.

Esta situación, en la que prevalece la no pertenencia a grandes consorcios internacionales de autopartes, hace que no se beneficien de contratos establecidos desde los países de origen con las plantas ensambladoras o con proveedores directos. Por tanto, se trata de empresas que se sitúan, en el mejor de los casos, en el segundo o tercer nivel de proveedores (proveedores o subproveedores de proveedores directos) y se dedican a la producción de partes de menor tecnología como soportes, resortes o al troquelado de piezas. Salvo las que exportan, el resto no tiene entregas JIT. Cabe mencionar que estas empresas, dada su fragilidad, han diversificado a sus clientes y realizan trabajos para otras ramas, como la de alimentos o la textil.

Como se puede observar, el proceso de reestructuración en la rama de autopartes está comandado por las empresas de mayor tamaño que son filiales de grandes consorcios mundiales; esta pertenencia les permite formar parte de encadenamientos globales, siguiendo estrategias de producción igualmente mundiales. Esto no quiere decir que se le niegue algún papel a la dinámica generada localmente, sino que se trata del predominio de vínculos de subordinación que responden a la lógica y al funcionamiento de estos grupos y al comportamiento del mercado exterior, el cual en la rama automotriz ha cobrado mayor relevancia que el interno.

La sola concentración en el espacio, y por ende su proximidad geográfica, no crean de manera automática o mecánica relaciones estrechas de cooperación y de intercambio de saberes adquiridos entre las empresas. Por lo menos en el caso de Querétaro, es claro que predomina una desarticula- 
ción local entre las empresas de la rama automotriz ya que en primer lugar su desarrollo no estuvo basado en una tradición industrial que ya existiera, sino que han sido resultado de lógicas y decisiones externas al territorio local; en segundo lugar no existe en el estado una planta armadora de automóviles, en torno de la cual pudiera constituirse un cluster automotriz, y en tercer lugar, el predominio de las empresas de capital extranjero hacen que pese más la lógica y las decisiones de los consorcios internacionales que operan con estrategias de producción globales, que la dinámica generada localmente. En este último punto se converge con lo planteado por Martinelli y Schoenberger (1994) en cuanto al papel desempeñado por los oligopolios en el proceso de reestructuración y al riesgo que corren las empresas más pequeñas de no poder resistir la competencia de las grandes empresas que se han fortalecido con grandes fusiones, como es el caso de la industria automotriz.

\section{Bibliografía}

Benko, Georges y Christophe Demazière (2000), "Le développement régional au miroir de la crise des régions d'ancienne tradition industrielle. L'exemple du nord de la France", en Georges Benko y Alain Lipietz (coords.), La Richesse des Régions, Presses Universitaires de France, París, pp. 451-478.

Benko, Georges y Alain Lipietz (2000), "Geographie socio-économique ou économie géographique?”, en Georges Benko y Alain Lipietz (coords.), La Richesse des Régions, Presses Universitaires de France, París, pp. 9-29.

CIEN (Centro de Información y Estudios Nacionales) (1992), “Querétaro industrial”, Voz Crítica, 5, Querétaro, pp. 20-31.

Estrada Correa, David Rafael (1996), "La industria en Querétaro”, en El águila se viste de oro, Canacintra Querétaro, Querétaro, pp. 27-44.

Garza, Gustavo (1985), El proceso de industrialización en la ciudad de México, 1821-1970, El Colegio de México, México.

Garza, Gustavo (1992), "Impacto regional de los parques industriales", Ciudades, 13, Red Nacional de Investigación Urbana, México, pp. 27-37. 
Gereffi, Gary y Miguel Korzeniewicz (1990), "Commodity Chains and Footwear Exports in the Semiperiphery", en William G. Martin (ed.), Semiperipherial States in the World Economy, Greenwood Press, Westport, Connecticut, pp. 45-68.

GEQ (Gobierno del Estado de Querétaro) (2004), La industria automotriz México-Querétaro, Secretaría de Desarrollo Sustentable-Dirección de Comercio y Estudios Económicos, Querétaro.

GEQ (Gobierno del Estado de Querétaro) (2009), Anuario Económico 2009, Secretaría de Desarrollo Sustentable, Querétaro, México.

GEQ (Gobierno del Estado de Querétaro) (2010), Industria automotriz en Querétaro, Secretaría de Desarrollo Sustentable, Querétaro.

Goldfrank, Walter L. (1995), "Beyond Cycles of Hegemony: Economic, Social, and Military Factors", Journal of World-Systems Research, 1 (8), Institute for Research on World-Systems-University of Utah-North Carolina State University, pp.1-14, <http:// jwsr.ucr. edu>, 23 de mayo de 2010 .

González Gómez, Ovidio y Rossana Nieto (2007), "Comportamiento logístico de las empresas de manufactura en Querétaro, México”, Economía, Sociedad y Territorio, vi (24), El Colegio Mexiquense, Zinacantepec, pp. 953-974.

González, Ovidio y Carmen Imelda González (1992), Transporte en Querétaro en el siglo XX, Secretaría de Comunicaciones y Transportes-Gobierno del Estado de Querétaro, México.

González Ovidio y Carlos Martner (1990), "Querétaro: ciudades fragmentadas", Ciudades, 6, Red Nacional de Investigación Urbana, México, pp. 23-30.

Hopkins, Terence K. e Immanuel Wallerstein (1986), "Commodity Chains in the World-Economy Prior to 1800”, Review, 10 (I), Binghamton University-State University of New York, Nueva York, pp. 157-170.

Hopkins, Terence K. e Immanuel Wallerstein (1994), "Commodity Chains in the Capitalist World-Economy Prior to 1800”, en Gary Gereffi 
y Miguel Korzeniewicz (eds.), Commodity Chains and Global Capitalism, Praeger, Westport, Connecticut, pp. 17-50.

INEGI (Instituto Nacional de Estadística, Geografía e Informática) (1994), XIV Censo Industrial 1994, INEGI, México.

INEGI (Instituto Nacional de Estadística, Geografía e Informática) (2002), La industria automotriz en México, INEGI, Aguascalientes.

INEGI (Instituto Nacional de Estadística, Geografía e Informática) (2004a), Censos Económicos, INEgi, México.

INEGI (Instituto Nacional de Estadística, Geografía e Informática) (2004b), Censos Industriales, INEGI, Aguascalientes.

INEGI (Instituto Nacional de Estadística, Geografía e Informática) (2006), La industria automotriz en México, INEGI, Aguascalientes.

INEGI (Instituto Nacional de Estadística, Geografía e Informática) (2009a), Censos Económicos, INEGI, Aguascalientes.

INEGI (Instituto Nacional de Estadística, Geografía e Informática) (2009b), Censos Industriales, INEGI, México.

Juárez Núñez, Huberto (2005), “La industria proveedora de autopartes”, en Huberto Juárez Núñez, Arturo Lara Rivero y Carmen Bueno Castellanos (coords.), El auto global. Desarrollo, competencia y cooperación en la industria del automóvil, Benemérita Universidad Autónoma de Puebla-Universidad Autónoma Metropolitana, Xochimilco-Universidad Iberoamericana-Consejo Nacional de Ciencia y Tecnología, México, pp. 71-116.

Kim, Hyung Kook y Su-Hoon Lee (1994), "Commodity Chains and the Korean Automobile Industry”, en Gary Gereffy y Miguel Korzeniewicz (eds.), Commodity Chains and Global Capitalism, Praeger, Westport, Connecticut, pp. 281-296.

Lee, Naeyoung y Jeffrey Cason (1994), "Automobile Commodity Chains in the NICs: A Comparison of South Korea, Mexico and Brazil", en Gary Gereffy y Miguel Korzeniewicz (eds.), Commodity Chains and Global Capitalism, Praeger, Westport, Connecticut, pp. 223-243. 
Lustig, Nora (1998), Mexico. The Remaking of an Economy, Brookings Institution Press, Washington.

Martinelli, Flavia y Erica Schoenberger (1994), "Los oligopolios están bien gracias. Elementos de reflexión sobre la acumulación flexible", en Georges Benko y Alain Lipietz (eds.), Las regiones que ganan, Alfons El Magnànim, Valencia, pp. 159-183.

Miranda, Eduardo (2005), Del Querétaro rural al industrial, 1940-1973, Universidad Autónoma de Querétaro-Miguel Ángel Porrúa, México.

Mortimore, Michael y Faustino Barron (2005), Informe sobre la industria automotriz mexicana, Comisión Económica para América Latina y el Caribe, Santiago de Chile.

Motta, Jorge, Mariela Cuttica y Leticia Zavaleta (2000), "Las relaciones proveedor-cliente en la industria automovilística argentina", Comercio Exterior, 50 (9), Banco Nacional de Comercio Exterior, México, pp. 820-829.

Novick, Marta y Gabriel Yoguel (2001), "La vulnerabilidad de una 'rama productiva': la difícil relación cliente-proveedor en el complejo automotriz argentino", Trabajo, 2a época, año 2, 4, Centro de Análisis del Trabajo, México, pp. 111-142.

SE (Secretaría de Economía) (2010), "Inversión extranjera directa por entidad federativa", <www.economia.gob.mx>, 26 de julio de 2010 .

Womack, James P., Daniel T. Jones y Daniel Roos (1992), La máquina que cambió al mundo, McGraw-Hill, Madrid.

Recibido: 21 de diciembre de 2008. Reenviado: 6 de diciembre de 2010. Aceptado: 18 de marzo de 2011.

Selva L. Daville-Landero. Es doctora en ciencias sociales por la Universidad Autónoma Metropolitana, Xochimilco; maestra en desarrollo urbano por El Colegio de México, y realizó sus estudios de licenciatura en economía en la Universidad Autónoma Metropolitana, Xochimilco. 
Desde 1992 es investigadora en el Centro de Investigaciones Interdisciplinarias en Ciencias y Humanidades de la Universidad Nacional Autónoma de México. Su línea de investigación es la reestructuración industrial y sus impactos territoriales. Entre sus publicaciones destacan: Querétaro: sociedad, economía, politica y cultura, CEIICH-UnAM, México (2000); "Conocimiento y aprendizaje para la democracia en las organizaciones sociales de Querétaro", en Jaime Castillo y Elsa Patiño (coords.), Saberes organizativos para la democracia, Red Nacional de Investigación Urbana-Universidad Autónoma de Puebla, México, pp. 119-136 (2001); "La trayectoria de industrialización de Querétaro", en Diana Villarreal, Dominique Mignot y Daniel Hiernaux (coords.), Dinámicas metropolitanas y estructuración territorial. Estudio comparativo México-Francia, UAM, Xochimilco-Miguel Ángel Porrúa, México, pp. 243-275 (2003); "Querétaro: un pôle émergent de développement industriel”, Revue d'Économie Régional et Urbaine, núm. esp., 5/2004, París, pp. 737-751 (2004); "Querétaro: un pôle émergent de développement industriel”, en Dominique Mignot y Diana Villarreal (coords.), Les Formes de la Métropolisation, Rapport No. 222, l'inrets, París, pp. 77-91 (2009). 